\title{
Decision making of a specialist Aortovascular multidisciplinary team meeting
}

\author{
Martin T. Yates ${ }^{\#}$, Ana Lopez-Marco ${ }^{\#}$, Michelle Lee, Benjamin Adams, John Yap, Rakesh Uppal, Aung Y. Oo \\ Department of Cardiothoracic Surgery, St Bartholomew's Hospital, London, UK \\ Contributions: (I) Conception and design: B Adams, J Yap, R Uppal, AY Oo; (II) Administrative support: None; (III) Provision of study materials or \\ patients: All authors; (IV) Collection and assembly of data: M Lee, MT Yates, A Lopez-Marco; (V) Data analysis and interpretation: MT Yates, A \\ Lopez-Marco; (VI) Manuscript writing: All authors; (VII) Final approval of manuscript: All authors. \\ "These authors contributed equally to this work as co-first authors. \\ Correspondence to: Miss Ana Lopez-Marco. Department of Cardiothoracic Surgery, St Bartholomew's Hospital, West Smithfield, EC1A 7BE, London, \\ UK. Email: ana.lopez-marco@nhs.net.
}

Background: Patients with major aortic pathology have complex needs, require lifelong surveillance and often multiple interventions. Multidisciplinary teams (MDT) have been shown to improve care in other specialities but there are no such reports related to dedicated Aortovascular MDTs. We aim to review our experience and decision making of an Aortovascular MDT over 5 years period since its beginning.

Methods: Cohort retrospective analysis of prospectively collected data for all patients referred to our Aortovascular MDT since its inception. Demographics, diagnosis and outcome of discussions were analysed.

Results: From April 2015 to April 2020, 871 discussions took place at the MDT meeting on 649 unique patients. Mean age was 52 [17-84] years, 414 (64\%) were male, $209(32 \%)$ had previous cardiac surgery and 85 (13\%) had connective tissue disorder. Final decision from the MDT meeting was surgery (220, 33.9\%), surveillance $(240,36.9 \%)$, not for intervention (42, 6.5\%), further investigation $(53,8.2 \%)$, assess for surgery $(30,4.6 \%)$, endovascular therapy $(28,4.3 \%)$, other $(36,5.5 \%)$. Of 649 patients discussed, $591(91 \%)$ underwent the management suggested by the meeting. Of the $58(9 \%)$ that were not carried out, the most common reason was patient preference.

Conclusions: Patients with major aortic pathology should expect care from a dedicated MDT, not only to benefit from the optimal choice of therapy but also the holistic care delivered by a team with expertise in all aspects of care for complex Aortovascular pathologies. We believe Aortovascular MDTs should become a standard of care in such patients.

Keywords: Aorta and great vessels; multidisciplinary team (MDT); clinical excellence; patient-based decision

Received: 07 June 2021; Accepted: 19 August 2021; Published: 30 March 2022.

doi: 10.21037/asj-21-47

View this article at: https://dx.doi.org/10.21037/asj-21-47

\section{Introduction}

Patients with major aortic pathology are complex and their diagnosis and management often bridge multiple specialities (1). Therefore, a coordinated team approach to their care would seem advisable, however dedicated multidisciplinary aortic teams are not a universal practise.
Multidisciplinary team (MDT) working is most commonly associated with oncology. Originally used in diagnostic decision making and standardized evidence-based treatment planning for patients with cancer, it has been shown to improve patient outcomes (2). Furthermore, there is evidence that its use, increases adherence to guidelines in individual patient's treatment (3).

$\wedge$ ORCID: 0000-0003-2313-2244. 
In cardiovascular disease, MDTs were originally used in the care of congenital heart disease and transplant patients. The Heart Team was first introduced to coronary revascularisation in clinical trials of angioplasty versus coronary artery bypass graft surgery. Since then, it has become an integral part of the pathway for patients with coronary artery and valvular heart disease (4-7). The success of the introduction and expansion of transcatheter aortic valve implantation (TAVI) procedure is the most striking example of the how effective the MDT meetingbased decision-making and MDT delivered care in clinical practice can be.

However, MDT meetings are not offered to patients with aortic disease world-wide, perhaps due to the presentation of these patients, often on an urgent or emergency basis, and also because open surgery remains the gold-standard treatment for most segments of the aorta. Moreover, many vascular and cardiothoracic units dealing with aortic disease do not have equally strong expertise in all treatment options (open surgery and endovascular therapy). This has led to a decision bias towards one or the other option which may not be the best for that particular patient.

In the United Kingdom, Aortovascular MDT meetings are common practice among most of the adult cardiac surgery units in the country, although its format varies significantly among regions, either in timing (from fixed weekly or bi-weekly sessions to ad-hoc discussions), in format (face to face meetings or videoconferences) and in representation of the specialities present at the discussions.

The Aortovascular Service at Barts Heart Centre began in 2015 after the merger of three Cardiothoracic Units (St Bartholomew's Hospital, London Chest Hospital and the University College - Heart Hospital). The Aortovascular MDTs were introduced at that point.

The surgical activity has exponentially increased since then, as well as the variety of aortic pathologies and the segments of the aorta treated. In 2017, the service incorporated an internationally recognised surgeon to expand the activity and to form the Complex Aortic Surgery unit, offering open surgical treatment of descending thoracic aorta and thoracoabdominal pathologies. Supported by the good results achieved, the number of referrals and the surgical activity increased exponentially, being currently the leading service in the United Kingdom. More recently, endovascular procedures have been also incorporated gradually to the service.

This study aims to review the demographics, decisionmaking and outcomes of the patients discussed at the
Aortovascular MDT.

We present the following article in accordance with the STROBE reporting checklist (available at https://asj. amegroups.com/article/view/10.21037/asj-21-47/rc).

\section{Methods}

The specialist Aortovascular MDT meeting was formed in Barts Heart Centre in 2015. The meeting occurs biweekly and is attended by cardiac surgery, congenital cardiac surgery, radiology, vascular and endovascular surgery, congenital cardiology and hypertension specialists. It is open to trainees in all specialties as well as other interested members of the healthcare team.

Referrals are accepted by email from any medical practitioner. The MDT is coordinated by the Aortic Surgery Fellow. Patient case history and imaging is reviewed and discussed. Outcomes are recorded contemporaneously in the patient's electronic records and maintained in a local MDT database. A designated clinical will be responsible for each patient decision.

We have conducted a descriptive retrospective review of prospectively collected data including all the discussions held at the Aortovascular MDT meeting from April 2015 until April 2020. We included demographics, primary diagnosis, referring team and the outcome clinical decision of the MDT. Secondary study points included compliance to the outcomes decisions and variability.

\section{Statistical analysis}

Results are presented as mean with range. Data and tables describe actual number with percentage in parentheses.

\section{Ethical statement}

This study was conducted in accordance with the Declaration of Helsinki (as revised 2013). The study was approved by institutional ethics board of Barts Heart Centre (ID 1118) and individual consent for this retrospective analysis was waived.

\section{Results}

\section{Demographics of patients discussed at MDT}

From April 2015 to April 2020, 871 discussions took place at the MDT meeting on 649 unique patients. Mean age 
Table 1 Primary aortic pathology at time of discussion at the Aortovascular multidisciplinary team meeting

\begin{tabular}{lc}
\hline Pathology & Number $(\%)(\mathrm{n}=649)$ \\
\hline Aortic valve disease & $54(8.3 \%)$ \\
Aneurysm & $341(52.7 \%)$ \\
Dissection & $109(16.8 \%)$ \\
Aneurysm + dissection & $55(8.5 \%)$ \\
Penetrating aortic ulcer & $20(3.1 \%)$ \\
Pseudoaneurysm & $18(2.8 \%)$ \\
Coarctation & $24(3.7 \%)$ \\
Endoleak/infection & $19(2.9 \%)$ \\
Other & $7(1.4 \%)$ \\
\hline
\end{tabular}

Table 2 Primary anatomical aortic segment affected at the time of discussion at the Aortovascular multidisciplinary team meeting

\begin{tabular}{lc}
\hline Aortic segment & Number $(\%)(\mathrm{n}=649)$ \\
\hline Aortic valve & $71(10.9 \%)$ \\
Aortic root & $134(20.6 \%)$ \\
Ascending aorta & $181(28 \%)$ \\
Ascending aorta + other & $14(2.1 \%)$ \\
Arch & $71(10.9 \%)$ \\
Descending aorta & $149(22.9 \%)$ \\
Abdominal aorta & $6(0.9 \%)$ \\
Peripheral artery & $23(23.5 \%)$ \\
\hline
\end{tabular}

was 52 [17-84] years and $414(64 \%)$ were male. A total of 209 patients (32\%) had previous cardiac surgery. Eighty patients $(13 \%)$ had a confirmed diagnosis of connective tissue disorder [Marfan's 48 (60\%); Turner's 20 (25\%), Loeys-Dietz 6 (7.5\%), Ehler-Danlos 5 (6.2\%), Williams 1 $(1.2 \%)]$ and 83 patients $(12.8 \%)$ had a confirmed bicuspid aortic valve. Timing of the referrals was: elective (567, $87.4 \%)$, urgent $(76,11.7 \%)$ or emergency $(6,0.9 \%)$.

\section{Pathology of patients discussed}

Primary pathology in these 649 patients was aneurysm (341, 52.7\%), aortic dissection (109, 16.8\%—of which, 42 (38.5\%) were type A (either chronic dissections or cases with residual distal dissection), 5 (4.6\%) none-A-none-B and $62(56.9 \%)$ were type $\mathrm{B}$ aortic dissections), aortic

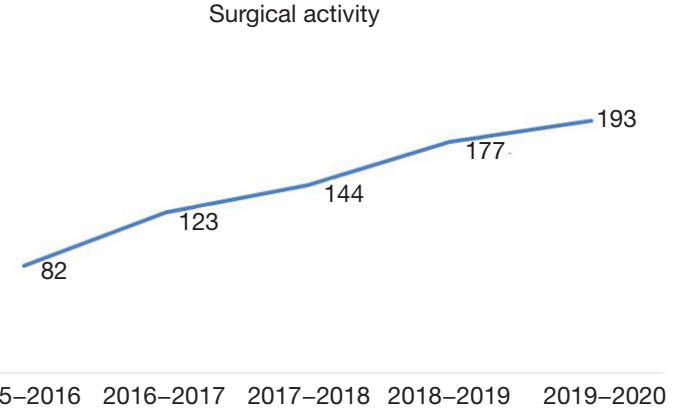

Figure 1 Display of the Aortovascular surgical activity (combined open and endovascular procedures) over time since creation of our institution. An increasing trend on surgical activity can be observed.

valve disease $(54,8.3 \%)$, aneurysm + dissection $(55,8.5 \%)$, penetrating aortic ulcer $(20,3.1 \%)$, pseudoaneurysm (18, $2.8 \%)$, coarctation $(24,3.7 \%)$, endoleak/infection $(19,2.9 \%)$ and other $(9,1.4 \%)$ (Table 1).

\section{Aortic segment of patients discussed}

For individual patients, of which there were 649, the main segment of aorta affected was aortic valve $(71,10.9 \%)$, aortic root $(134,20.6 \%)$, ascending aorta $(181,28 \%)$, ascending aortic + other (14, 2.1\%), arch (71, 10.9\%), descending aorta $(149,22.9 \%)$, abdominal aorta $(6,0.9 \%)$ or peripheral artery $(23,3.5 \%)$ (Table 2$)$.

\section{Referrals over time}

The number of referrals per year has increased over time reflecting the expansion of the Aortovascular centre in our institution, with the incorporation of surgical expertise to treat complex aortic pathologies. We discussed 124 patients $(14.2 \%)$ in the first year, $161(18.5 \%)$ in the second year, $174(19.9 \%)$ third year, $218(25.0 \%)$ fourth year and $194(22.2 \%)$ last year. This trend is matched by the Aortovascular surgical activity over the study time period with the total number of cases increasing from 82 to 193 per year (Figure 1). The complexity of procedures performed also increased in this time period (Figure 2).

\section{Referring teams}

The majority of the patients for MDT discussions were referred by Cardiothoracic Surgery (304, 46.8\%), followed 
Surgical procedures

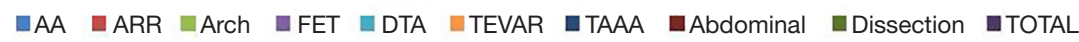

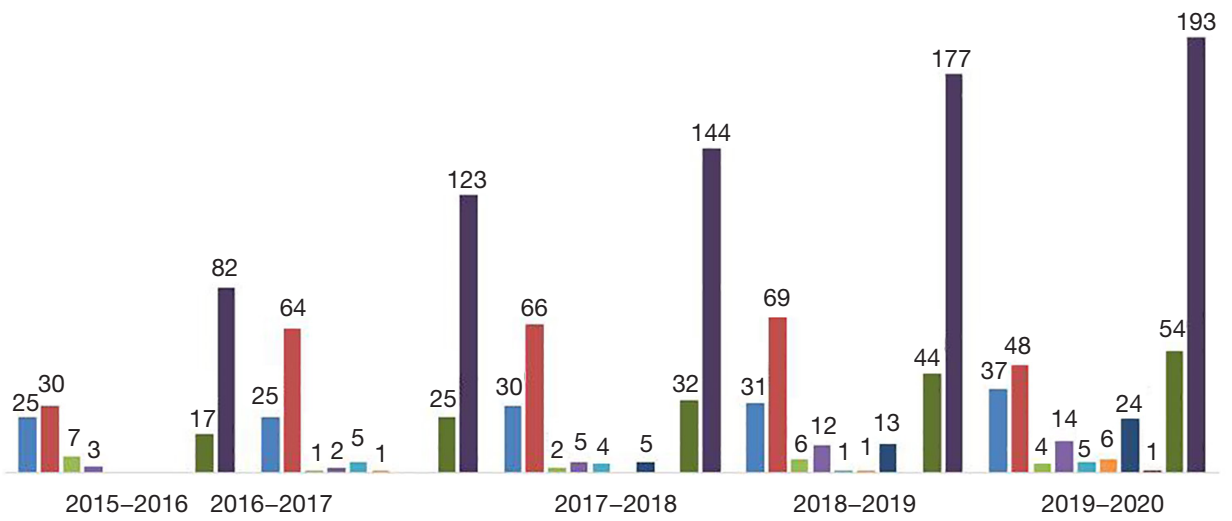

Figure 2 Representation of the variety of Aortovascular surgical procedures performed in our unit each year. We can observe an increase in the total number of procedures performed each year, as well as the progressive introduction of complex distal Aortovascular procedures (both open and endovascular). AA, ascending aorta replacement; ARR, aortic root replacement; FET, frozen elephant trunk procedure; DTA, descending thoracic aorta replacement; TEVAR, thoracic endovascular aortic replacement; TAAA, open thoracoabdominal aortic repair.

Table 3 Decision outcomes reached after presentation and discussion at the Aortovascular Multidisciplinary Team (MDT) Meeting

\begin{tabular}{lc}
\hline MDT outcome & Number of patients $(\%)(\mathrm{n}=649)$ \\
\hline Surgery & $220(33.9 \%)$ \\
Surveillance & $240(36.9 \%)$ \\
Not for intervention & $42(6.5 \%)$ \\
Further investigations & $53(8.2 \%)$ \\
Assess for surgery & $30(4.6 \%)$ \\
Endovascular therapy & $28(4.3 \%)$ \\
Other & $36(5.5 \%)$ \\
\hline
\end{tabular}

in decreasing incidence by: Grown Up Congenital Heart (GUCH) services $(195,30.0 \%)$, Vascular Surgery (49, 7.5\%), Adult Cardiology (46, 7.1\%), General Practitioners $(7,1.1 \%)$ and Other medical specialties (6, 0.9\%). In 42 cases $(6.5 \%)$ the referring team was not recorded.

\section{Geographical origin of patients discussed}

We assessed the distance from patients' home to our centre for a representative consecutive selection of 399 patients from August 2017 to June 2019. 205 (51\%) were from within a 10 -mile radius, $133(33 \%)$ were from $10-50$ miles and $46(12 \%)$ were from more than 50 miles away and $5(1 \%)$ of these being outside the country.

\section{Decision making and implementation of MDT decisions}

Final decision from the MDT meeting was surgery (220, $33.9 \%)$, surveillance (240, 36.9\%), not for intervention (42, $6.5 \%)$, further investigation (53, 8.2\%), assess for surgery (30, 4.6\%), endovascular therapy $(28,4.3 \%)$, other (36, $5.5 \%$ ) (Table 3).

Of 649 patients discussed, 591 (91\%) underwent the management suggested by the meeting. Of the 58 (9\%) that were not carried out, the reasons in decreasing order were: patient preference $(16,27.6 \%)$, individual surgeon's decision $(14,24.1 \%)$, need for urgent surgery due to clinical progression $(9,15.5 \%)$, change on the treatment modality to a less invasive option $(6,10.3 \%)$, patient died waiting for treatment $(5,8.6 \%)$, patient was deemed not fit for the intervention suggested $(4,6.9 \%)$ and delay in planned outcome $(4,6.9 \%)$.

The decision outcomes made by the MDT were more commonly followed in patients referred by other teams (Adult Cardiology, GUCH and Vascular) than in patients added by the local Cardiothoracic team-compliance 91.3- 
91.8\% vs. $87.8 \%(\mathrm{P}<0.001)$.

Decision outcomes were more commonly followed in emergency $(100 \%)$ and urgent $(90.8 \%)$ discussions compared to elective cases $(82.9 \%)(\mathrm{P}<0.001)$.

\section{Discussion}

Aortic diseases have different clinical presentation patterns - a congenital disorder in childhood, an incidental finding during screening or investigation of other symptoms or as an emergency. Whether managed surgically or conservatively, patients require lifelong surveillance in a dedicated Aortovascular clinic and may undergo multiple procedures, both open surgical and endovascular (1). They are likely to have pathology affecting multiple segments of the aorta and its branches. Treatment strategies must include planning for subsequent interventions and the optimal timing and order in which these should be carried out. Furthermore, specialist assessment and testing for inherited conditions and risk factor management such as hypertension are essential to improve long term outcomes before and after intervention. There is also a need for thorough counselling of patients and family for lifestyle modification, reassurance, family planning and psychosocial support to achieve the optimal lifelong quality outcomes.

With such a variety of presentations, patients' initial interaction with health care can be from a wide range of specialities. The speciality that they see first can influence their subsequent management due to selection bias and local expertise and knowledge. Such practice is known to occur in coronary disease with patients more likely to undergo PCI when presenting to hospitals without in house cardiac surgery.

Coordinated care of patients with complex lifelong needs is essential and can be best managed by a specialist Aortovascular MDT. This must include all members of the team involved in patient care. Comprising, but not be limited to cardiologist, cardiac and vascular surgeon, radiologist, anaesthetist, clinical geneticist, clinical psychologist and hypertension specialist. These meetings should occur on a regular basis, at a convenient time and with sufficient time for discussions. A dedicated room should be available with adequate IT support and preferably a video link to allow outside institutions to join in (8). All discussions should be documented and fed back to the referring physician in an appropriate time and the system should be audited for quality control. Existing guidelines certainly form the basis for our decisions based on size criteria, growth and risk factors and at the most basic level it could be argued this negates the need for an MDT. The MDT is useful for more complex patients who have additional factors not discussed in guidelines, have particular patient preference, have had previous intervention affecting future decisions or current decision making will guide or influence future interventions. This is similar to PCI vs. CABG in coronary MDTs which also have very clear guidelines however discussions are often interesting and useful despite this.

MDT discussions promote effective team working, with the patients benefiting from a patient-centred-care covering various aspects of care and preventing treatment decision bias from a single clinical specialty, which may not be in the best interest of patient. All of our aortic surgeons attend the meeting and each has their specialist interest or skill. General cardiac surgeons will refer patients to the group for consideration of specialist surgery such as valve sparing root or arch replacement. Amongst the aortic surgeons, part of the MDT process is to decide which surgeon (or two together) will operate on the patient, depending on the surgery that is required. A further benefit is that shared decision making pre operatively encourages shared periand post-operative care of every aortic patient which we hope also improves outcomes.

On the other hand, there are also disadvantages associated with MDT meetings, such as lack of ownership of the patient and conviction for patients, possible less than ideal decisions due to lack of adequate/firsthand information about patients (e.g., decision based on presented information rather than first-hand clinical assessment), potential for not reaching decision despite multiple discussions due to lack of adequate time for discussion and analysis of information, and potential abuse of the MDT meeting process to turn down perceived high risk patients.

Assessing the effectiveness of an MDT is difficult and this is reflected in the fact that there is little literature in the cardiovascular field to support its use. The ESC/EACTS Guidelines included the importance of MDT-Heart Team discussions in pathologies like infective endocarditis, complex coronary artery disease and high-risk aortic and mitral valve disease, however this is based on expert opinion rather than research evidence (4-6). It is often impossible to identify patients not discussed at an MDT as a control population, those discussed at an MDT are often more complex and might be a component of selection and/or decision bias $(8,9)$. 
Currently the most reliable indicator of a functioning MDT is the percentage of decisions carried out. Several scoring tools for MDT have been proposed however they are complex and require independent observation of the meetings (10). Other important domains that reflect the quality of the MDT are the prioritisation of complex cases, the wide representation of all specialities involved and a balance between quality of the discussions and the duration of process and the times from discussion to the delivery of the suggested outcome. Once a decision is reached, the duty of the MDT is not over as the patient will require clinic assessment followed by the delivery of the chosen treatment and subsequent period of surveillance. Patient preference is, of course paramount, and should be taken on account when analysing the effectiveness of these discussions, as one of the most common reason for MDT decisions not being carried out is patient preference.

Our results have shown that since the Aortovascular MDT meetings were established, the number of patients referred to the meeting for discussion has increased gradually, expanding the referring teams as well as the referring geographical areas. In addition to new referrals, patients will be discussed multiple times as their condition changes over the years. This trend further increases the teams experience and ability to management those patients with the most complex aortic pathologies.

Limitations of the study include the descriptive nature of the data and the difficulty of identifying a control population group of similar patients who are not discussed at an MDT to establish variability in outcomes. This may initiate the interest and ability to perform a trial to answer this question, which individual centres will find impossible to perform. Moreover, the fact that our Aortovascular MDT was created by the cardiac surgical team, might explain why patients referred from other specialties achieve a decision outcome (open aortic surgery in the majority of the cases) more frequently than patients referred from our own team. Growth in the number of patients discussed over time does not mean that actual patient care is better. We use the increase in volume, not as a surrogate of improved patient outcomes, but is certainly a reflection that both referring teams and the MDT itself find the process useful.

\section{Conclusions}

Patients with major aortic pathology should expect care from a dedicated MDT, not only to benefit from the optimal choice of therapy but also the holistic care delivered by a team with expertise in all aspects of care for complex Aortovascular pathologies. We believe Aortovascular MDTs should become a standard of care in such patients and the process should be audited regularly to guarantee adhesion to the outcomes decisions on a timely manner.

\section{Acknowledgments}

This paper was presented at the Houston Aortic Symposium on the $7^{\text {th }}$ March 2020 in Houston (Texas, USA).

Funding: None.

\section{Footnote}

Reporting Checklist: The authors have completed the STROBE reporting checklist. Available at https://asj. amegroups.com/article/view/10.21037/asj-21-47/rc

Data Sharing Statement: Available at https://asj.amegroups. com/article/view/10.21037/asj-21-47/dss

Conflicts of Interest: All authors have completed the ICMJE uniform disclosure form (available at https://asj.amegroups. com/article/view/10.21037/asj-21-47/coif). AYO serves as an unpaid editorial board member of AME Surgical fournal from April 2021 to March 2023. The other authors have no conflicts of interest to declare.

Ethical Statement: The authors are accountable for all aspects of the work in ensuring that questions related to the accuracy or integrity of any part of the work are appropriately investigated and resolved. The study was conducted in accordance with the Declaration of Helsinki (as revised in 2013). The study was approved by institutional ethics board of Barts Heart Centre (ID 11118) and individual consent for this retrospective analysis was waived.

Open Access Statement: This is an Open Access article distributed in accordance with the Creative Commons Attribution-NonCommercial-NoDerivs 4.0 International License (CC BY-NC-ND 4.0), which permits the noncommercial replication and distribution of the article with the strict proviso that no changes or edits are made and the original work is properly cited (including links to both the formal publication through the relevant 
DOI and the license). See: https://creativecommons.org/ licenses/by-nc-nd/4.0/.

\section{References}

1. Erbel R, Aboyans V, Boileau C, et al. 2014 ESC Guidelines on the diagnosis and treatment of aortic diseases:

Document covering acute and chronic aortic diseases of the thoracic and abdominal aorta of the adult. The Task Force for the Diagnosis and Treatment of Aortic Diseases of the European Society of Cardiology (ESC). Eur Heart J 2014;35:2873-926.

2. Kesson EM, Allardice GM, George WD, et al. Effects of multidisciplinary team working on breast cancer survival: retrospective, comparative, interventional cohort study of 13,722 women. BMJ 2012;344:e2718.

3. Ray-Coquard I, Thiesse P, Ranchère-Vince D, et al. Conformity to clinical practice guidelines, multidisciplinary management and outcome of treatment for soft tissue sarcomas. Ann Oncol 2004;15:307-15.

4. Neumann FJ, Sousa-Uva M, Ahlsson A, et al. 2018 ESC/ EACTS Guidelines on myocardial revascularization. Eur Heart J 2019;40:87-165.

5. Baumgartner H, Falk V, Bax JJ, et al. 2017 ESC/EACTS Guidelines for the management of valvular heart disease.

doi: 10.21037/asj-21-47

Cite this article as: Yates MT, Lopez-Marco A, Lee M, Adams B, Yap J, Uppal R, Oo AY. Decision making of a specialist Aortovascular multidisciplinary team meeting. AME Surg J 2022;2:2.
Eur Heart J 2017;38:2739-91.

6. Habib G, Lancellotti P, Antunes MJ, et al. 2015 ESC

Guidelines for the management of infective endocarditis: The Task Force for the Management of Infective Endocarditis of the European Society of Cardiology (ESC). Endorsed by: European Association for Cardio-Thoracic Surgery (EACTS), the European Association of Nuclear Medicine (EANM). Eur Heart J 2015;36:3075-128.

7. Head SJ, Kaul S, Mack MJ, et al. The rationale for Heart Team decision-making for patients with stable, complex coronary artery disease. Eur Heart J 2013;34:2510-8.

8. Pavlidis AN, Perera D, Karamasis GV, et al. Implementation and consistency of Heart Team decisionmaking in complex coronary revascularisation. Int J Cardiol 2016;206:37-41.

9. Yates MT, Soppa GK, Valencia O, et al. Impact of European Society of Cardiology and European Association for Cardiothoracic Surgery Guidelines on Myocardial Revascularization on the activity of percutaneous coronary intervention and coronary artery bypass graft surgery for stable coronary artery disease. J Thorac Cardiovasc Surg 2014;147:606-10.

10. Taylor C, Atkins L, Richardson A, et al. Measuring the quality of MDT working: an observational approach. BMC Cancer 2012;12:202. 